\title{
Realization of Three-dimensional Walking of a Cheetah-modeled Bio-inspired Quadruped Robot
}

\author{
Shogo Nakatsu, Andre Rosendo, Masahiro Shimizu and Koh Hosoda
}

\begin{abstract}
Adaptability of quadruped animals is not solely reached by brain control, but by the interaction between its body, environment, and control. Especially, morphology of the body is supposed to contribute largely to the adaptability. We have tried to understand quadrupedal locomotion by building a bio-inspired quadruped robot named "Pneupard", which has a feline-like muscular-skeletal structure. In our previous study, we successfully realized alternative gait of hindlimbs by reflex control based on the sole touch information, which is called an unloading rule, and that of forelimbs as well. In this paper, we finally connect forelimbs and hindlimbs by a rigid spine, and conduct 3D walking experiments only with the simple unloading rule. Through several preliminary experiments, we realize that the touch information on the sole is the most critical for stable 3D walking.
\end{abstract}

\section{INTRODUCTION}

Quadruped animals can adaptively locomote over a variety of terrain. Such adaptability is not solely reached by brain control, but by interaction between body, environment, and brain [1], [2]. Many researchers studied about quadruped animals. They observed and measured motions of the animals, and recorded myoelectric activities to explain the functions of the muscles. However, they could not give any definite answer for explaining the adaptability. There may be ethical problems that arise when we apply physically or electrically interfere to living animals for investigating function of the body parts. To avoid such problems, the constructive approach draws the attention, in which we develop a bio-inspired robot, experiment on it, and identify functions of the body.

Fukuoka et al. [3] developed a quadruped robot using DC motors. They controlled the robot by a CPG model based on a neural oscillator which proposed by Taga et al. [4]. In addition, they realized three kinds of reflex control on the robot. They successfully made the robot walk on irregular terrains and suggested importance of an interaction between CPGs and reflex sensory feedback control. Yamada et al. [5] developed a bio-inspired quadruped robot which has 20 pneumatic artificial muscles and springs and investigated about the importance of bi-articular muscles in galloping

This work was partially supported by Grant-in-Aid for Scientific Research on 23220004, 25540117 of Japan.

Shogo Nakatsu and Andre Rosendo are with Graduate School of Information Science and Technology, Osaka University, 2-1 Yamadaoka, Suita, Osaka, 565-0871, Japan. Masahiro Shimizu and Koh Hosoda are with Graduate School of Engineering Science, Osaka University, 1-3 Machikaneyama, Toyonaka, Osaka, 560-8531, Japan. nakatsu.shougodist.osaka-u.ac.jp

andre.rosendo@ist.osaka-u.ac.jp

shimizu@sys.es.osaka-u.ac.jp

hosodalsys.es.osaka-u.ac.jp gait. Ekeberg et al. [6] presented a robot which duplicated the muscular-skeletal systems of a cat's hindlimb in the simulation and succeeded to it walk by using simple control. These work gave us a lot of insight on adaptability of quadrupedal locomotion, but to identify more definite functions of the muscles, we need more precise copy of the muscular-skeletal system.

For investigating mechanism from the constructive approach, we develop a bio-inspired quadruped robot named "Pneupard". We adopt a cheetah as a model and try to duplicate its muscular-skeletal structure as much as possible by utilizing pneumatic artificial muscles. It has 4 links and 9 muscles in each forelimb and 3 links and 7 muscles in each hindlimb. In our previous studies [7], [8], we focused on only hindlimbs or forelimbs and proposed a sole reflexbased control system. First, we built a Pneupard's hindlimbs and successfully made the robot walk on a treadmill [7]. Next, we built Pneupard's forelimbs and succeeded to make the robot walk on a treadmill [8].

In this paper, we connect the hindlimbs and forelimbs by a rigid spine and attempt to make the robot walk by the reflex-based control. Through the rigid spine, the legs are interacting with each other, but we do not apply any extrinsic communication among legs: only physical interaction through the rigid spine. In our previous work [7], [8], the robot only had hindlimbs or forelimbs, therefore, the movement was almost in the sagittal plane. By combining the legs, the robot can now move in $3 \mathrm{D}$, which makes its locomotion more challenging. We basically apply the same reflex-based control as [7] and [8], and show how it works through real experiments. Owaki et al. [9] adopted similar control to successfully make a simple quadruped robot walk. From their success, though their robot is much smaller and simpler, we expect the robot walk, and as a result, we can investigate the functions of the muscles.

The paper is organized as follows. First, we depict the design of Pneupard and the reflex-based control system. Then, we show some 3D walking experimental results with the robot. Finally, we discuss our results and present future work.

\section{Robot DESIGN}

For investigating the interaction between a body of the quadruped animal and the environment, we built a bioinspired quadruped robot named "Pneupard" modeling a cheetah (Figure 1). Lengths of the links, range of joints, and holdfasts of muscles are determined based on the observation of the animal, by Hudson et al. [10], [11], Done et al. [12], 


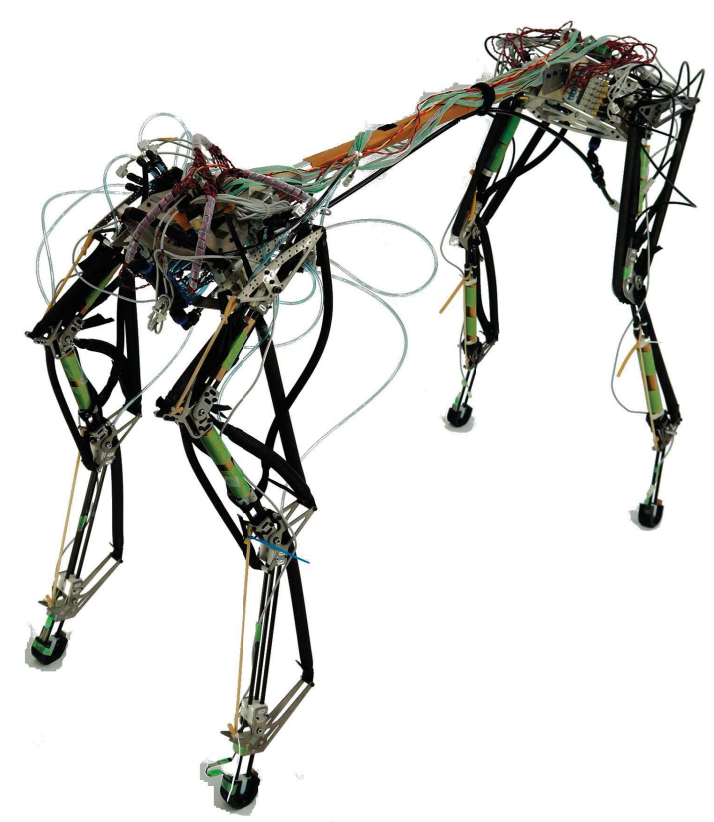

Fig. 1. The design of a bio-inspired quadruped robot named "Pneupard" modeling a cheetah. Pneupard is actuated by Mckibben pneumatic artificial muscles. The size of the robot is similar to an adult cheetah. This robot's spine has no DOF, and is not flexible. Pneupard has 18 DOF and 38 muscles including the muscles for adduction/abduction.

and Goslow et al. [13]. We paid attention to design moment arm of each muscle trying to mimic the real cheetah's structure as much as possible. Figure 2 shows comparison between moment arms of a real cheetah and those of the robot. Muscle lengths are determined by considering the range of motion of the joint. This robot is $1.7 \mathrm{~m}$ length, $0.3 \mathrm{~m}$ width, $0.63 \mathrm{~m}$ height, and about $7 \mathrm{~kg}$ weight.

Pneupard is actuated by Mckibben pneumatic artificial muscles which enables us to build animal-like muscularskeletal structure. We produced all the pneumatic artificial muscles, and the contracting ratio is up to $24 \%$. When pressured air is supplied into a pneumatic muscle, it contracts and its compliance increases. If the air is exhausted, it gets back the original length and its compliance decreases.

Figure 3 shows joints and muscles of Pneupard. It has totally 18 DOF: 5 DOF in each forelimb - adduction/abduction, scapula, shoulder, elbow, and wrist - and 4 DOF in each hindlimb - adduction/abduction, hip, knee and ankle (the adduction/abduction joint is not depicted in the figure). The forelimb has 8 pneumatic artificial muscles and 1 spring muscle: 5 mono-articular muscles, Atlantoscapularis (AS), supraspinatus (SS), pectoralis major (PM), triceps brachii(lateral and medial head) (TBlm), and flexor carpi ulnaris (FCU), and 4 bi-articular muscles, Latissimus dorsi (LD), triceps brachii(long head) (TB), biceps brachii (BB), and extensor carpi radialis (ECR). The hindlimb has 7 pneumatic artificial muscles and 1 spring muscle: 5 monoarticular muscles, Iliopsoas (IL), biceps femoris (BF), vastus lateralis (VL), soleus (SO), and tibias anterior (TA), and 3 bi-articular muscles, Rectus femoris (RF), semimembranosus

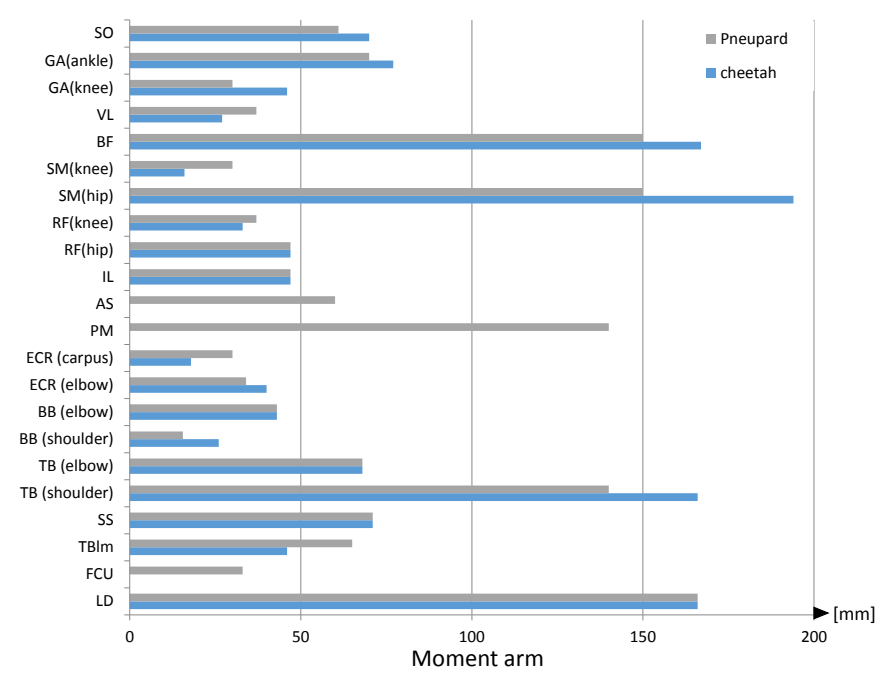

Fig. 2. Graph of comparison between moment arms of a real cheetah and those of Pneupard. To the best of the author's knowledge, the moment arm of some muscles are not mentioned anywhere. We decide their muscles' moment arm reffered by muscles' arrangement or range of motion of joints.

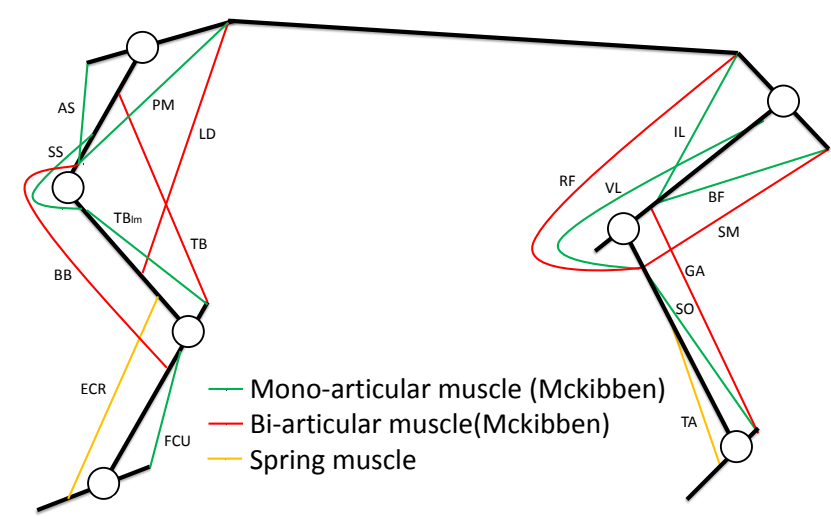

Fig. 3. Joints and muscles of Pneupard. Each forelimb has 4 DOF, 4 biarticular muscles and 5 mono-articular muscles. Each hindlimb has 3 DOF, 3 bi-articular muscles and 5 mono-articular muscles. Spine has no DOF. Biarticular pneumatic muscles are depicted in green. Mono-articular pneumatic muscles are depicted in red. Spring muscles are depicted in yellow.

(SM), and gastrocnemius (GA). Although biceps femoris is a bi-articular muscle, its moment arm at the knee joint is so small that we can functionally suppose it as a mono-articular muscle. In total, Pneupard has 38 muscles including the muscles for adduction/abduction. Functions of the muscles are shown in Table I. Gray boxes indicate bi-articular muscles.

The pressure of the air source is regulated as $0.7 \mathrm{MPa}$. We can measure pressure of pneumatic artificial muscles by sensors (PSE540). The air is supplied to the muscles through on-off magnetic valves (VQZ series). Each foot of the robot has a force sensing register (FSR406) to observe ground reaction force in real time.

\section{CONTROL SYSTEM}

Each leg is controlled independently by a state machine. A gait cycle of a leg is divided into 4 states : touchdown, stance, lift-off, and swing. A transition from one state to the 
TABLE I

FUNCTIONS OF MUSCLES

\begin{tabular}{|l|l|}
\hline muscle & Functions of muscles \\
\hline Atlantoscapularis & scapula flexion \\
Supraspinatus & shoulder flexion \\
Pectralis Major & scapula extension \\
Triceps Brachii(lateral,medial) & elbow extension \\
Flexor Carpi Ulnaris & wrist extension \\
Latissimus Dorsi & scapula extension, shoulder flexion \\
Triceps Brachii(long) & shoulder flexion, elbow extension \\
Biceps Brachii & shoulder extension, elbow flexion \\
Extensor Carpi Radialis & elbow flexion, wrist flexion \\
Iliopsoas & hip flexion \\
Biceps Femoris & hip extension \\
Vastus Lateralis & knee extension \\
Soleus & ankle extension \\
Tibias Anterior & ankle flexion \\
Rectus Femoris & hip flexion, knee flexion \\
Semimembranosus & hip extension, knee extension \\
Gastrocnemius & knee extension, ankle extension \\
\hline
\end{tabular}

other is triggered by signals of the force sensing register in the sole (touchdown, stance, and lift-off) or by time (swing). Figures 4 and 5 show the state transitions of the forelimb and hindlimb, respectively. Movement of the leg in each state is determined by a desired pressure pattern of the muscles, which is designed by referring real EMG patterns observed in the real animals [14], [15]. The desired pressure pattern used for the experiments is shown in Table II. We modify some muscles' activities not to follow the real EMG pattern so that the movement of the robot does not become too large. In the table, desired pressures in gray boxes are not necessarily determined by the real data, but are determined by trial and error.

TABLE II

DESIRED PRESSURE PATTERN OF THE MUSCLES [MPa].

\begin{tabular}{|c|c|c|c|c|}
\hline FORELIMB & Swing & Touchdown & Stance & Liftoff \\
\hline FCU & 0.0 & 0.52 & 0.72 & 0.72 \\
TB & $0.36-0.0$ & 0.36 & 0.36 & 0.36 \\
BB & $0.36-0.72$ & 0.0 & 0.0 & 0.36 \\
PM & 0.0 & 0.0 & $0.0-0.72$ & 0.36 \\
LD & 0.36 & 0.36 & 0.36 & 0.36 \\
TBlm & 0.52 & 0.52 & 0.52 & 0.52 \\
SS & 0.72 & 0.72 & 0.72 & 0.72 \\
AS & 0.72 & 0.72 & 0.72 & 0.72 \\
\hline
\end{tabular}

\begin{tabular}{|c|c|c|c|c|}
\hline HINDLIMB & Swing & Touchdown & Stance & Liftoff \\
\hline SO & 0.091 & 0.52 & 0.52 & 0.52 \\
GA & 0.091 & 0.52 & 0.52 & 0.52 \\
VL & 0.0 & 0.52 & 0.67 & 0.67 \\
SM & 0.61 & 0.46 & 0.30 & 0.72 \\
BF & 0.061 & 0.061 & $0.061-0.52$ & 0.061 \\
RF & 0.42 & 0.42 & $0.52-0.72$ & 0.67 \\
IL & 0.72 & 0.72 & 0.72 & 0.72 \\
\hline
\end{tabular}

The state machine switches from swing to touchdown automatically after a fixed period of time $\left(T_{\text {swing }}\right)$. In the touchdown state, atlantoscapularis, supraspinatus, triceps brachii(long head), triceps brachii(lateral and medial head), latissimus dorsi, and flexor carpi ulnaris are activated in the forelimb. In the hindlimb, soleus, gastrocnemius, vastus lateralis, rectus femoris, semimembranosus, and iliopsoas are activated. Swiching from touchdown to stance is triggered when the ground reaction force exceeds a threshold $\left(F_{S T}\right)$. In the forelimb, atlantoscapularis, pectralis major, supraspinatus, triceps brachii(long head), triceps brachii(lateral and medial head), latissimus dorsi, and flexor carpi ulnaris are activated, and soleus, gastrocnemius, vastus lateralis, rectus femoris, biceps femoris, semimembranosus, and iliopsoas are activated in the hindlimb. Then, the state machine switches from stance to lift-off when the ground reaction force becomes sufficiently low $\left(F_{L O}\right)$. Atlantoscapularis, pectralis major, supraspinatus, triceps brachii(long head), triceps brachii(lateral and medial head), latissimus dorsi, biceps brachii, and flexor carpi ulnaris are activated in the forelimb. Soleus, vastus lateralis, rectus femoris, semimembranosus, and iliopsoas are activated in the hindlimb. Finally, switching from lift-off to swing is triggered when the foot leaves the ground, that is, the ground reaction force is below a threshold $F_{S W}$. Atlantoscapularis, supraspinatus, triceps brachii(lateral and medial head), latissimus dorsi and biceps brachii are activated in the forelimb, and rectus femoris, semimembranosus, and iliopsoas are activated in the hindlimb in the swing state. This state machine is a realization of a unloading rule originally proposed by Ekeberg et al. [6], but is modified for real muscular-skeletal legs.

Each pneumatic muscle is pressure-controlled to follow the desired pressure indicated in Table II. In this paper, the adduction/abduction joint is not controlled, but is fixed physically, therefore, each leg moves in the sagittal plane.

There is no explicit communication between legs here. However, the legs are connected by a rigid spine, we can expect the physical interaction among them. When the body (the torso) is moving forward, we can expect ground reaction force of one of the hindlimbs eventually decreases, and it will be in the lift-off state. After the leg moves forward and touches down, another hindlimb is expected to be in lift-off state. The lift-off of the forelimb is even more complicated : since the legs are connected by a spine, it may swing back and forth, and the forelimb may lose reaction force. Since the robot has four legs, one of them always lifts up. If a pattern of the swing legs emerges appropriately, the robot may walk only by the reaction-based control, which may be one of the most fundamental walking controllers. In ants, Cruse [16] found similar mechanism to generate leg coordination without global communication. Owaki et al. [9] investigated similar leg coordination in a quadruped. Since the legs of the quadruped were simple and position-controlled, they could use phase. In our muscular-skeletal quadruped, we could not simply apply phase because each leg is too complecated. For utilizing the structured compliance of the leg, we apply a state machine.

\section{WALKING EXPERIMENT AND RESULT}

We conduct a walking experiment on the real robot to check the ability of the independent unloading rule to each joint. In each experimental trial, the experimenter holds the robot spine, make it move, and after a while, he/she release the robot. 


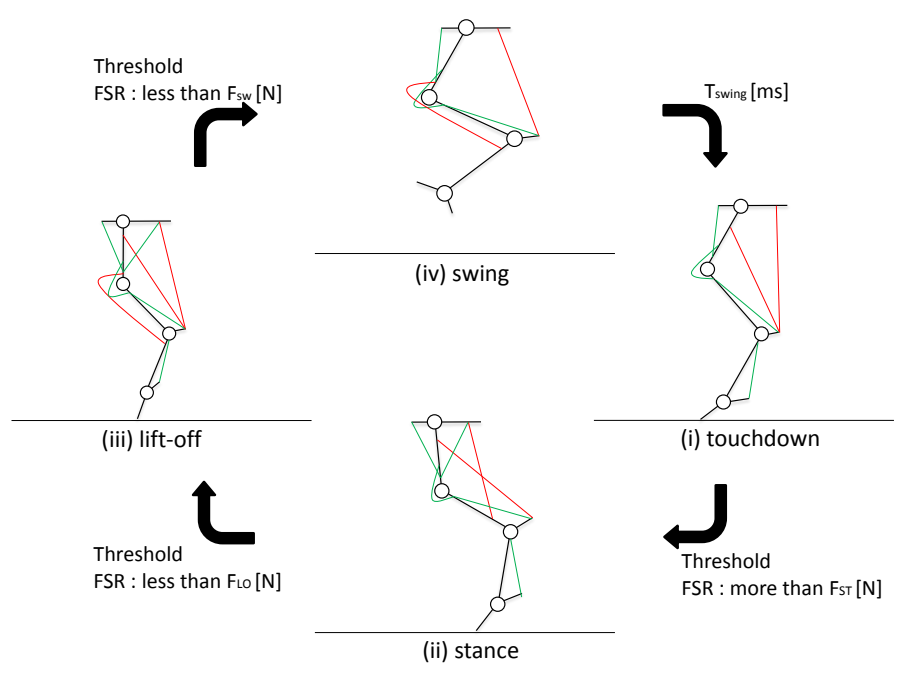

Fig. 4. The state transition for a walking motion of the forelimb. Leg control system is separated for each leg, and at any point in time, it is in 1 state of 4 states : touchdown, stance, lift-off, and swing. A transition from one state to the other is triggered by signals of the force sensing register in the sole (touchdown, stance, and lift-off) or by time (swing).

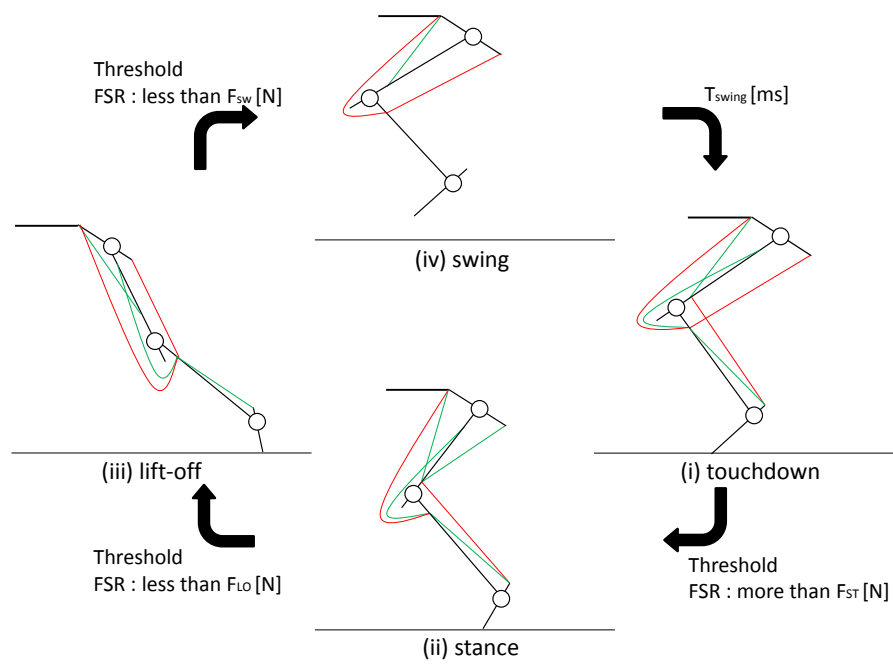

Fig. 5. The state transition for a walking motion of the hindlimb. Leg control system is the same as that of the forelimb.

Figure 7 shows one successful walking motion sequence of the robot, and Figure 6 shows the gait diagram of walking at the trial. When the ground reaction force is above a threshold, which means stance, the output is 1 , otherwise the output is 0 . From top to bottom, the lines indicate states of left hindlimb (RL), right hindlimb (RR), left forelimb (FL), and right forelimb (FR), respectively.

One walking cycle of a leg takes about 3 seconds. As we can see in the diagram, from $500 \mathrm{~ms}$ to $3000 \mathrm{~ms}$, the robot exhibit a walking motion pattern. First, incidentally, the state of the right forelimb changed to lift-off, and then to swing. After the right forelimb touched the ground, the state of the left forelimb changed to lift-off, and then to swing. This sequence might be the result of the rolling motion of the

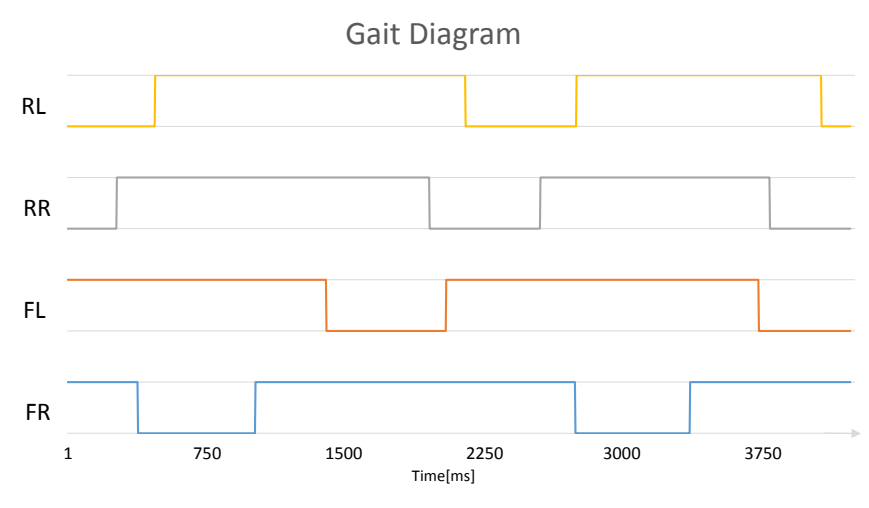

Fig. 6. Graph of the gait diagram. When the leg touches the ground, which means stance and lift-off state, the output is 1 , otherwise the output is 0 . From top to bottom, the lines indicate states of left hindlimb (RL), right hindlimb (RR), left forelimb (FL), and right forelimb (FR), respectively.

torso. Before swing of the left forelimb changed to stance, the ground reaction force of the right hindlimb decreased and its state changed to swing. Finally, the state of the left hindlimb changed to lift-off and then to swing before that of the right hindlimb changed to stance.

\section{DiscusSION}

In this paper, we demonstrate that a muscular-skeletal robot, Pneupard, controlled by an unloading rule can emerge walking gait pattern even if it does not have any global communication among leg controllers. The walking emerges from the interaction among body, brain (control) and environment. As already pointed out, Cruse [16] found similar mechanism to generate leg coordination without global communication. Owaki et al. [9] investigated similar leg coordination in a quadruped. Our point in this paper, is that the robot has complicated muscular-skeletal structure, and we should have used a state machine to control the robot instead of utilizing phase. However, we can share the idea that the physical body can play a role of communication. The emerged gait pattern was a typical walking pattern, which was our surprise.

The emerging gait pattern of Pneupard is, however, not a typical walking pattern : front right leg - rear left leg - front left leg - rear right leg. In typical walking gait pattern, a kittycornered hindlimb begin moving after a forelimb moves. However, the Pneupard's walking pattern is different. Maybe, the emerging gait pattern depends on plural factors such as body speed, elasticity of spine and muscles, flexibility of joints, so on.

In the future, we plan to give a muscle activation pattern for a big walking motion to Pneupard. We will activate all muscles and succeed to make Pneupard walk and express the general gait pattern. Next, we will give a flexible spine to Pneupard. Eventually, we want to investigate the gait transition by using Pnaupard. 


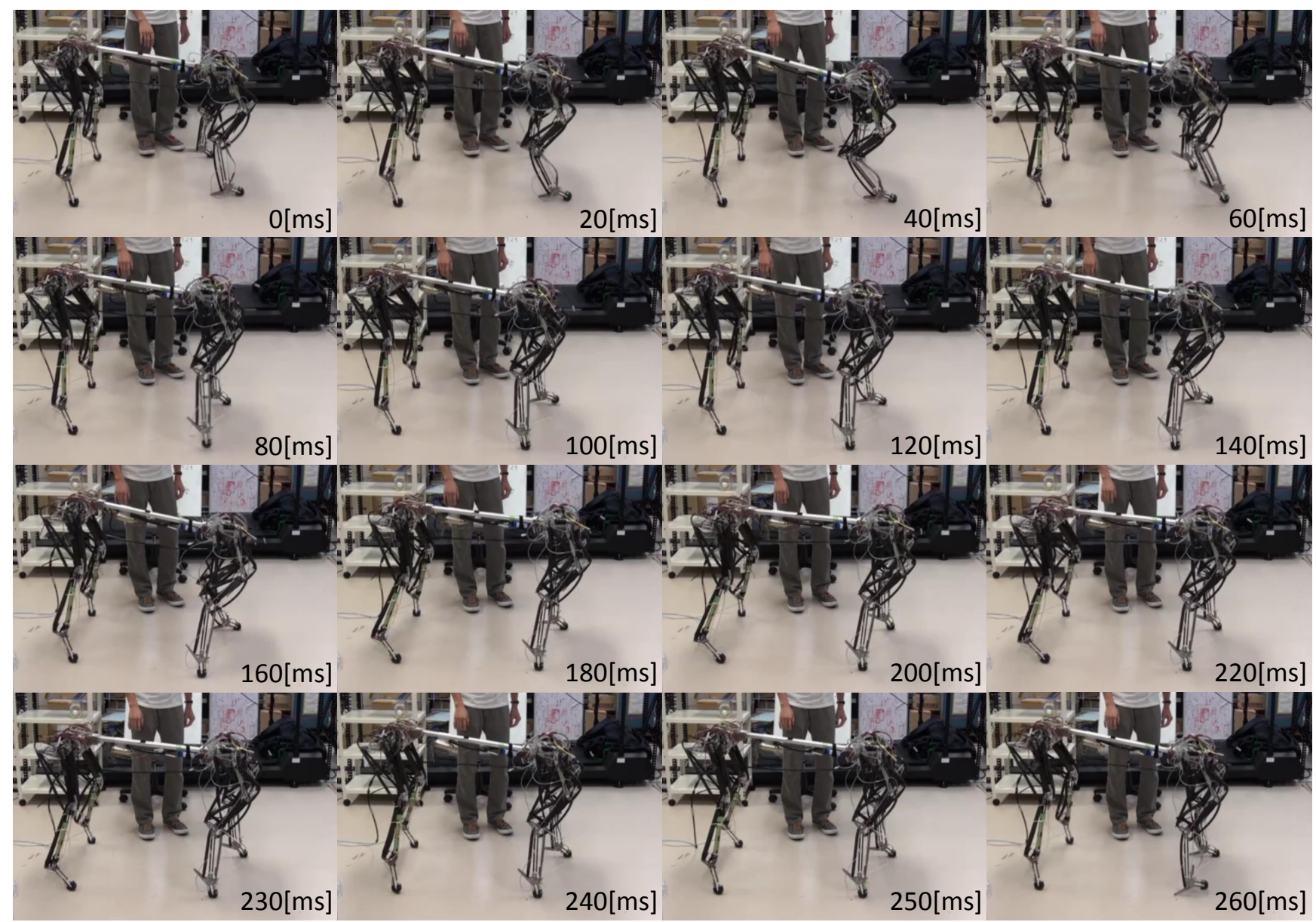

Fig. 7. One successful walking motion sequence of the robot. One walking step takes about 3 seconds. This shows the procession of one walking step cycle. The front right leg moves in a period from $0 \mathrm{~ms}$ to $100 \mathrm{~ms}$. The front left leg moves in a period from $120 \mathrm{~ms}$ to $180 \mathrm{~ms}$. The rear right leg moves in a period from $180 \mathrm{~ms}$ to $240 \mathrm{~ms}$. The rear left leg moves in a period from $230 \mathrm{~ms}$ to $260 \mathrm{~ms}$.

\section{ACKNOWLEDGEMENT}

The authors would like to thank Dr. Kenichi Narioka of research institute for cognition and robotics, Bielefeld University. The authors would like to thank a specially assistant professor Shuhei Ikemoto of institute for academic initiatives, Osaka University.

\section{REFERENCES}

[1] Rodney A. Brooks. Intelligence without representation. Journal of Artificial Intelligence, September 1987.

[2] Hiroshi Kimura, Isao Shimoyama, and Hirofumi Miura. Dynamics and control of the quadruped robot(dynamic walk by use of the redundancy of actuators caused by the multiple touching legs). The Japan Society of Mechanical Engineers (C part) , 55(517):2396-2401, Sep. 1989.

[3] Yasuhiro Fukuoka and Hiroshi Kimura. Biologically inspired adaptive dynamic walking of a quadruped on irregular terrain -realization of walking in outdoor environment using a self-contained robot: "tekken2"-. JRSJ, 25:138-154, 2007.

[4] G.Taga, Y.Yamaguchi, and H.Shimizu. Self-organized control of bipedal locomotion by neural oscillators in unpredictable environment. Journal of Biological Cybernetics, 65:147-159, 1991.

[5] Yasunori Yamada, Satoshi Nishikawa, Kazuya Shida, Ryuma Niiyama, and Yasuo Kuniyoshi. Neural-body coupling for emergent locomotion: A musculoskeletal quadruped robot with spinobulbar model. In IROS'11, pages 1499-1506, 2011.
[6] Orjan Ekeberg and Keir Pearson. Computer simulation of stepping in the hind legs of the cat: An examination of mechanisms regulating the stance-to-swing transition. Journal of Neurophysiology, 94:42564268, 2005.

[7] Andre Rosendo, Shogo Nakatsu, Kenichi Narioka, and Koh Hosoda. Pneupard: A biomimetic musculoskeletal approach for a felineinspired quadruped robot. In IROS, pages 1452-1457, 2013.

[8] Shogo Nakatsu, Andre Rosendo, Kenichi Narioka, and Koh Hosoda. Stable reflex-based walking of forelimbs of a bio-inspired quadruped robot-modeled cheetah. In ROBIO, pages 1813-1818, 2013.

[9] Dai Owaki, Takeshi Kano, Ko Nagasawa, Atsushi Tero, and Akio Ishiguro. Simple robot suggests physical interlimb communication is essential for quadruped walking. The Royal Society, 10, Janually 2013.

[10] Penny E. Hudson, Sandra A. Corr, Rachel C. Payne-Davis, Sinead N. Clancy, Emily Lane, and Alan M. Wilson. Functional anatomy of the cheetah (acinonyx jubatus) forelimb. Journal of Anatomy, 218:375385, 2011.

[11] Penny E. Hudson, Sandra A. Corr, Rachel C. Payne-Davis, Sinead N. Clancy, Emily Lane, and Alan M. Wilson. Functional anatomy of the cheetah (acinonyx jubatus) hindlimb. Journal of Anatomy, 218:363374, 2011.

[12] Stanley H. Done, Peter C. Goody, Susan A. Evans, and Neil C. Stickland. Color Atras of Veterinary Anatomy The Dog and Cat. MOSBY ELSEVIER, 2009.

[13] George E. Goslow, Robert M. Reinking JR., and Douglas G. Stuart. The cat step cycle: Hind limb joint angles and muscle lengths during unrestrained locomotion. In Journal of Morphology, volume 141, pages $1-42,2005$.

[14] Arthur William English. An electromygraphic analysis of forelimb 
muscles during overground stepping in the cat. In Journal of Experimental Biology, volume 76, pages 105-122, October 1978.

[15] I.Engberg and A.Lundberg. An electromyographic analysis of muscular activity in the hindlimb of the cat during unrestrained locomotion. Acta Physiologica Scandinavica, 75:614-630, 1969.

[16] Holk Cruse. What mechanisms coordinate leg movement in walking arthropods? Trends in Neurosciences, 13:15-21, 1990. 